\title{
Absolute thickness imaging of PMMA layer on corrugated transparent substrate by scanning white light interference microscopy.
}

\author{
Marc Jobin and Raphael Foschia
}

Ecole d'Ingénieurs de Genève, University of Applied Science, 4 rue de la Prairie, CH-1202 Genève, SWITZERLAND

NanoFeel, 4 Tir-au-Canon, CH-1227 Carouge, SWITZERLAND

Thickness measurement of transparent layer is usually performed either with mechanical profilers stylus or Atomic Force Microscope- on step edges or with optical techniques such as ellipsometry or white light reflectometry. Common drawbacks of those approaches include, for profilers, the necessity to have a step edge and registry with the substrate and, for optical techniques, the lack of spatial resolution or extreme dependence on refractive index.

Much attention has been devoted over the past 3 years to use scanning white light interference microscopy to have access to the absolute thickness of transparent layers [1]. Most of the time, the (semi)transparent layer is deposited onto a flat and reflecting surface, such as silicon wafer. We have used a home-made scanning white light interference microscope [2] depicted in Fig. 1a) to perform non-contact and absolute thickness imaging of PMMA (polymethylmetacrylate) layer over a second PMMA layer, i.e over a non flat and very low reflecting surface. We used two successive layers of PMMA spin coated at a very low speed $(60 \mathrm{rpm})$ on silicon wafer (Fig 1b). PMMA has been chosen for its widespread use in semiconductor and MEMS industry. Letting the first layer dried before spin coating of the second PMMA layer ensured sufficient reflectivity at the PMMA/PMMA interface.

White-light interference microscopy in the vertical scanning mode gives access to the true height information through the deconvolution of the envelope of the correlogram. In case of semitransparent material deposited on reflecting substrate or interface, each interface (PMMA/PMMA and PMMA/air in our case) will produce a fringe pattern as the Mirau objective is vertically scanned though the interfaces, as shown in Fig. 1c). The resulting correlograms on each pixel therefore has the shape of a double peak, which contains the absolute height information of both interfaces (Fig.2), and therefore of the thickness. On the correlogram shown in Fig.2, the optical thickness $L$ ' was 7.42um, which gave a true layer thickness $L=L^{\prime} / n=4.97 \mathrm{um}(n=1.492$ for PMMA). To have precise values of the position of the interface, a modified center-of-mass algorithm has been used.

Repeating the procedure for each pixel allows representing the topography of both air/PMMA and PMMA/PMMA interface. A 3D view of the PMMA/PMMA interface is shown in Fig 3a) and the absolute thickness on a plane section is shown in Fig 3b)

The technique has been successfully used as well for systems such as single acrylic thick film deposited on rough silver substrate.

\section{References}

[1] P. de Groot and X. Colonna, Appl. Opt., 43, 4821 (2004).

[2] M. Jobin, A. Du and R. Foschia, Proc. NSTI-Nanotech, 2, 695 (2005) 
a)

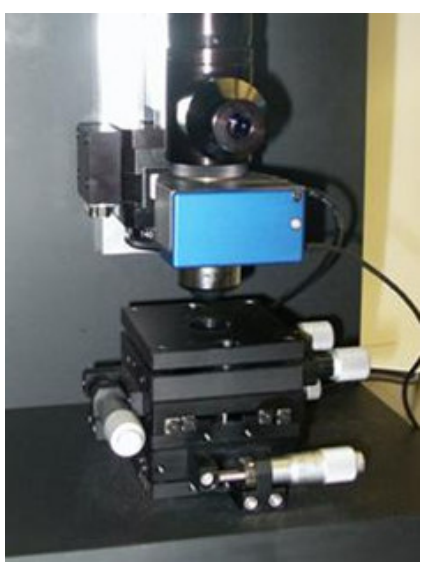

b)

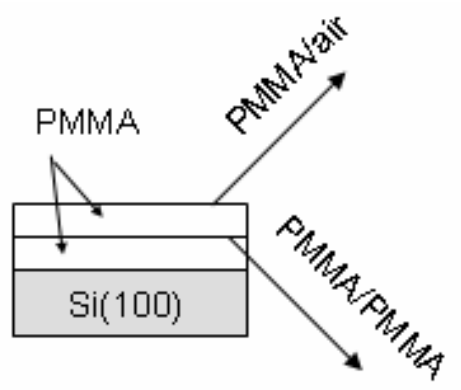

c)

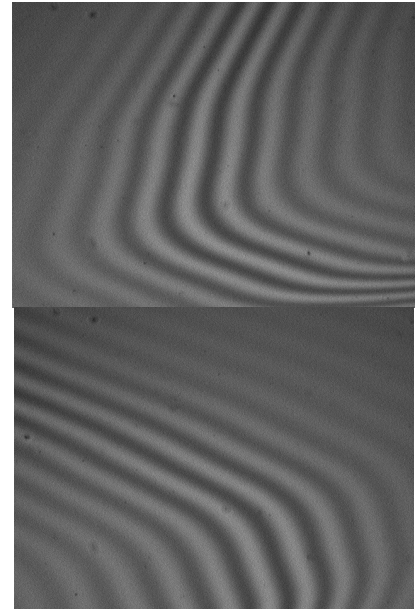

FIG. 1. a) Setup of the optical profiler. b) Sample description c) Interferogram over the PMMA/air interface (top) and the PMMA/PMMA interface (bottom). Field of view : $180 \mathrm{um}$ x $130 \mathrm{um}$

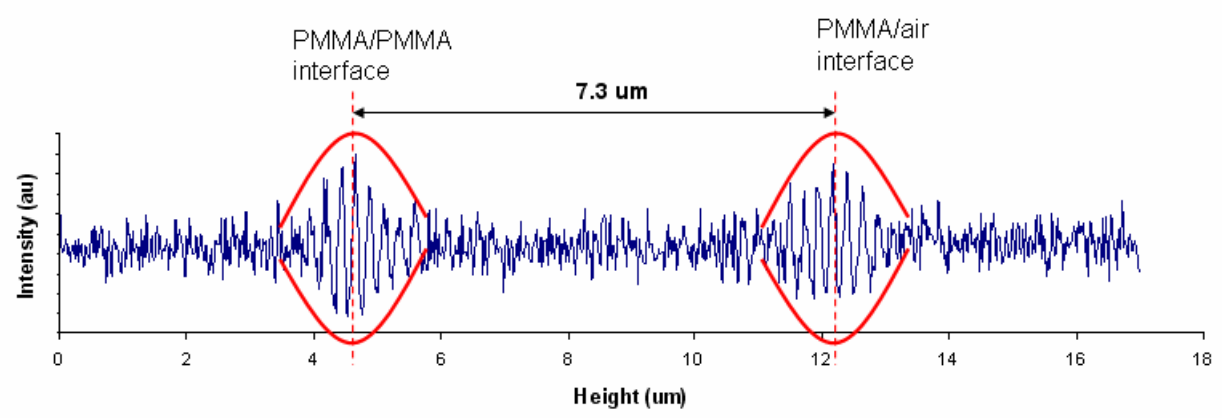

FIG. 2. Raw white-light interference correlogram of PMMA/PMMA/Si, showing the double peak of the envelope and then the optical thickness of the layer.
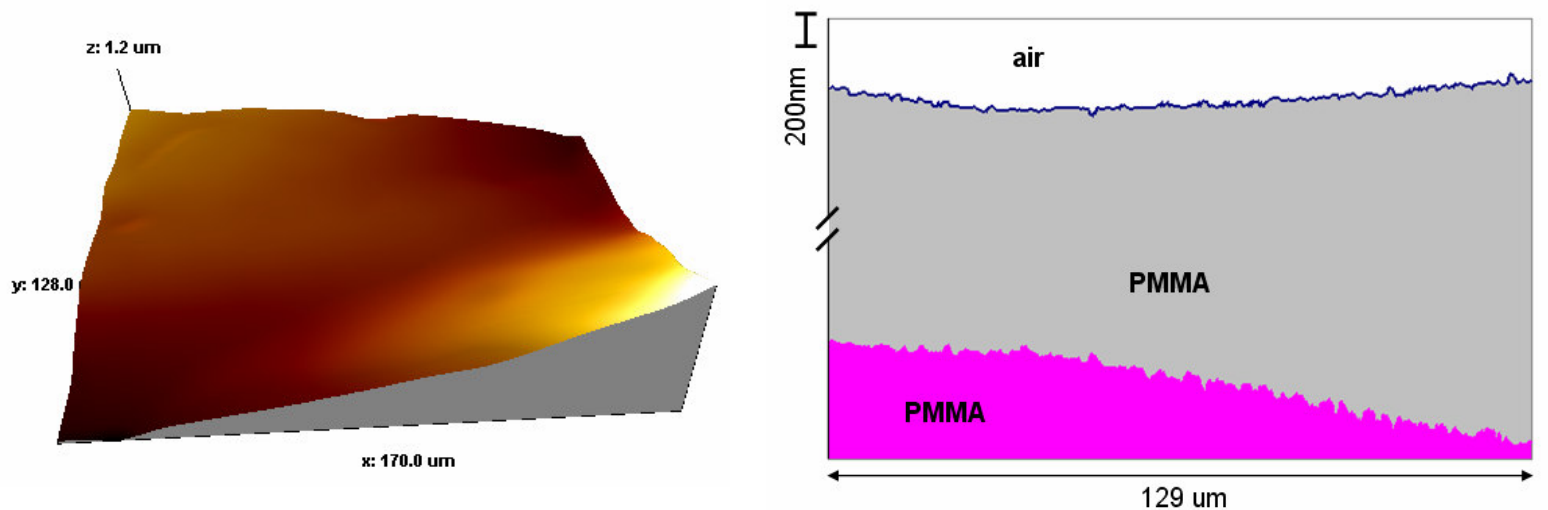

FIG. 3. 3D reconstruction of the PMMA/PMMA interfaces (left) and lane cross section showing the two interfaces (right) 